\title{
Infinite disorder scaling of random quantum magnets in three and higher dimensions
}

\author{
István A. Kovács ${ }^{1,2, * \text { and Ferenc Iglói }}{ }^{2,3, \oplus}$ \\ ${ }^{1}$ Department of Physics, Loránd Eötvös University, \\ H-1117 Budapest, Pázmány P. s. 1/A, Hungary \\ ${ }^{2}$ Research Institute for Solid State Physics and Optics, H-1525 Budapest, P.O.Box 49, Hungary \\ ${ }^{3}$ Institute of Theoretical Physics, Szeged University, H-6720 Szeged, Hungary
}

(Dated: June 24, 2018)

\begin{abstract}
Using a very efficient numerical algorithm of the strong disorder renormalization group method we have extended the investigations about the critical behavior of the random transverse-field Ising model in three and four dimensions, as well as for Erdős-Rényi random graphs, which represent infinite dimensional lattices. In all studied cases an infinite disorder quantum critical point is identified, which ensures that the applied method is asymptotically correct and the calculated critical exponents tend to the exact values for large scales. We have found that the critical exponents are independent of the form of (ferromagnetic) disorder and they vary smoothly with the dimensionality.
\end{abstract}

\section{INTRODUCTION}

Quantum phase transitions are among the fundamental problems of modern physics, the properties of which are studied in solid state physics, quantum field-theory, quantum information and statistical mechanics ${ }^{1}$. These transitions take place at $T=0$ temperature, i.e. in the ground state of the quantum system by varying a control parameter, such as the strength of a transverse field. One basic question in this field of research is how quenched disorder influences the properties of quantum phases and phase transitions. In this respect quantum spin glasses and the glass transition are particularly interesting ${ }^{2}$. This latter problem theoretically is very challenging, since the corresponding quantum state is the result of an interplay between quantum and disorder fluctuations, strong correlations and frustration.

One of the paradigmatic models of random quantum magnets with a discrete symmetry is the random transverse-field Ising model (RTIM), which is defined by the Hamiltonian:

$$
\mathcal{H}=-\sum_{\langle i j\rangle} J_{i j} \sigma_{i}^{x} \sigma_{j}^{x}-\sum_{i} h_{i} \sigma_{i}^{z} .
$$

Here the $\sigma_{i}^{x, z}$ are Pauli-matrices and $i, j$ denote sites of a lattice (or a graph). Experimentally the RTIM is closely related to the compound ${ }^{3} \mathrm{LiHo}_{\mathrm{x}} \mathrm{Y}_{1-\mathrm{x}} \mathrm{F}_{4}$, in which there is a dipole-coupling between the Ising spins, thus the interaction is long-ranged. Applying a magnetic field $H_{t}$ transverse to the Ising axis results in a transverse field of strength, $h_{i}=H_{t}^{2}$, but this transverse field induces a random longitudinal field $\underline{\underline{4}}$ via the off-diagonal terms of the dipolar interaction. In the theoretical investigations the interactions in the RTIM are generally assumed to be short-ranged, thus the first sum in Eq.(11) runs over nearest neighbors. Furthermore the $J_{i j}$ couplings and the $h_{i}$ transverse fields are independent random numbers, which are taken from the distributions, $p(J)$ and $q(h)$, respectively. For random ferromagnets we have $J>0$, whereas for spin-glasses there are both ferro- and antiferromagnetic couplings. Here we are basically interested in the former problem.

Detailed theoretical results about the RTIM are known in one dimension (1D) due to a complete analytical solution of a renormalization group (RG) treatment $\mathrm{t}^{-}$. The RG results are expected to be asymptotically exact in the vicinity of the critical point (and also in the Griffithsphase, as long as dynamical singularities are concerned ${ }^{6}$ ), which is indeed demonstrated by a comparison with independent analytical $\underline{7.8}$ and numerical ${ }^{9,10}$ works. One important observation, that the critical properties of the 1D model are governed by an infinite disorder fixed point (IDFP), in which the strength of disorder growths without limit during renormalization 11 and thus become dominant over quantum fluctuations.

The IDFP scenario is found to be valid for the 2D RTIM, too, as observed in numerical RG studies $12-18$ and in Monte Carlo (MC) simulations $\frac{19}{}$. The calculated critical exponents are in agreement with the MC results about the $2 \mathrm{D}$ random contact process 20 , which is a simple nonequilibrium model of spreading infections. The $d$-dimensional random contact process is expected to be in the same universality class 21 as the RTIM, at least for strong enough disorder.

In three dimensions, which is connected to real quantum magnets, no quantitative results are known, so far. Analysis of the numerical RG trajectories lead to the conclusion $\frac{12}{2}$, that the critical behavior in this case is probably controlled by an IDFP, but no estimates about the critical exponents are available. For even higher dimensions it is unclear, if the IDFP scenario stays valid for any finite value of $d$, or there is some upper critical dimension, $d^{c}$, so that for $d>d^{c}$ the critical behavior is of conventional disorder type. We note that the large- $d$ limit of the problem is qualitatively relevant for models with long-range interactions. In this respect the critical behavior of the (non-random) $\mathrm{LiHoF}_{4}$ system has been the subject of intensive MC simulations ${ }^{22}$.

In this paper we extend the investigations about the critical behavior of the RTIM into the hitherto unexplored three and higher dimensions. Here we have developed a considerably improved numerical algorithm of the strong disorder RG (SDRG) procedure and study large 
samples with $N \gtrsim 10^{6}$ sites. In $3 \mathrm{D}$ and $4 \mathrm{D}$ we consider hypercubic lattices and we study the large-D limit of the problem, too. This latter is realized by Erdős-Rényi (ER) random graphs ${ }^{23}$ consisting of $N$ sites and $k N / 2$ edges $(k>2)$, which are at random positions. The sizes of the largest systems we studied are shown in Table \. We note that the numerical algorithm of the SDRG method is completely different from that used in the $2 \mathrm{D}$ case, however, the steps used to calculate the critical parameters as well as the method of analysing the results are similar to that used in $2 \mathrm{D}^{\underline{18}}$.

The structure of our paper is the following. The SDRG method and its improved algorithm used in this paper is described in Sec II Results about the critical parameters are calculated in Sec III and discussed in Sec IV]

\section{SDRG PROCEDURE}

In the calculation we used the SDRG procedure 24 , which has been introduced by Ma, Dasgupta and $\mathrm{Hu}^{25}$. In this method, which works for random ferromagnets, at each step of the renormalization the largest local term in the Hamiltonan (either a coupling or a transverse field) is eliminated and new terms are generated between remaining sites by second-order perturbation method. After decimating a strong coupling, say $J_{i j}$, the two connected spins form a spin cluster having an additive moment, $\tilde{\mu}=\mu_{i}+\mu_{j}$, which is placed in an effective transverse field of strength: $\tilde{h}=\frac{h_{i} h_{j}}{J_{i j}}$. After decimating a large transverse field, $h_{i}$, the actual spin is eliminated and new effective couplings are generated between each pair of spins being nearest neighbors to the decimated site, say $j$ and $k$, having a value: $\tilde{J}_{j k}=\frac{J_{j i} J_{i k}}{h_{i}}$. If at one step two parallel couplings appear between two neighboring sites the maximum of them is taken. Application of this "maximum rule" is exact at an IDFP and results in simplifications of the RG procedure.

Here we have developed an optimized algorithm, which needs $t \sim \mathcal{O}(N \log N+E)$ time to renormalize a cluster with $N$ sites and $E$ edges up to the last spin, irrespective of the dimension and topology of the cluster $\underline{26}$. In this algorithm terms in the Hamiltonian are decimated in descending order in energy and we have applied the following theorem for transverse field decimation. According to this theorem for a decimated site, $i$, there is always one relevant neighboring site, $j$, so that after decimating $i$ only those renormalized couplings should be created, which connect $j$ with its new neighboring sites 27 . All the couplings which start from other nearest neighbors of $i$ (and does not end at $j$ ) are irrelevant and need not be created. In this way during one RG step not only the number of sites is reduced (by one), but the number of couplings, as well. Using this algorithm we avoid to generate almost fully connected clusters, which is the main drawback of the naïve implementation of the method in higher dimensions $\frac{18}{18}$, having a performance: $t \sim \mathcal{O}\left(N^{3}\right)$. Renormalization with the naïve and the improved algorithms is illustrated in Fig 1

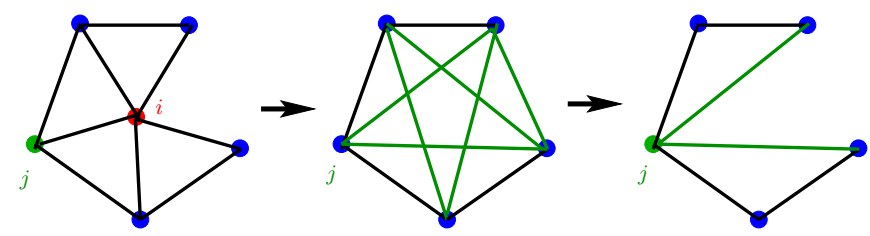

FIG. 1: (Color online) Illustration of the renormalization procedure. In the original cluster (left panel) the central site, $i$, is to be decimated. In the naïve implementation (middle panel) several new couplings are generated. In the improved algorithm (right panel) just the couplings to the relevant site, $j$, are created.

\section{CALCULATION OF CRITICAL PARAMETERS}

In the actual calculation - in order to check universality and to control the disorder dependence of the estimates - we have used two different forms of randomness. Both have the same uniform distribution of the couplings: $p(J)=\Theta(J) \Theta(1-J)(\Theta(x)$ being the Heaviside step-function), which are ferromagnetic. For the 'box- $h$ ' disorder also the transverse fields are uniformly distributed: $q(h)=\frac{1}{h_{b}} \Theta(h) \Theta\left(h_{b}-h\right)$, whereas for the 'fixed- $h$ ' model we have a constant transverse field 28 : $h_{i}=h_{f}$. We used the logarithmic variable, $\theta=\ln \left(h_{b}\right)$ or $\theta=\ln \left(h_{f}\right)$, as a quantum control parameter. We have checked that the computational time to renormalize an $N=10^{3}\left(N=10^{6}\right)$ cluster is typically $\sim 0.015(\sim 50)$ second (in a $2.4 \mathrm{GHz}$ processor), which does not depend on the dimension and the topology of the cluster. The numbers of realizations used in the calculations were typically 40000 but even for the largest sizes we have at least 10000 samples.

In the first step of the calculation for each random sample, $\alpha$, we have determined a pseudo-critical point, $\theta_{c}(\alpha, N)$, by a variant of the doubling method. In this procedure $\underline{\underline{18}}$ we glue together two identical copies $\left(\alpha, \alpha^{\prime}\right)$ of the sample by surface couplings 29 and renormalize it up to the last site for different values of the control parameter, $\theta$. The renormalization is found to be qualitatively different for $\theta<\theta_{c}(\alpha, N)$ and for $\theta>\theta_{c}(\alpha, N)$. For weak quantum fluctuations, $\theta<\theta_{c}(\alpha, N)$, the last decimated spin cluster contains equivalent sites of $\alpha$ and $\alpha^{\prime}$. These sites and thus the two replicas are correlated and we call this cluster as a correlation cluster. The moment of the correlation cluster, $\mu(\alpha, N)$, goes to zero as the pseudo-critical point is approached. On the contrary for $\theta>\theta_{c}(\alpha, N)$ in the last decimated spin cluster there are no equivalent sites of $\alpha$ and $\alpha^{\prime}$ and thus there is no correlation cluster. 


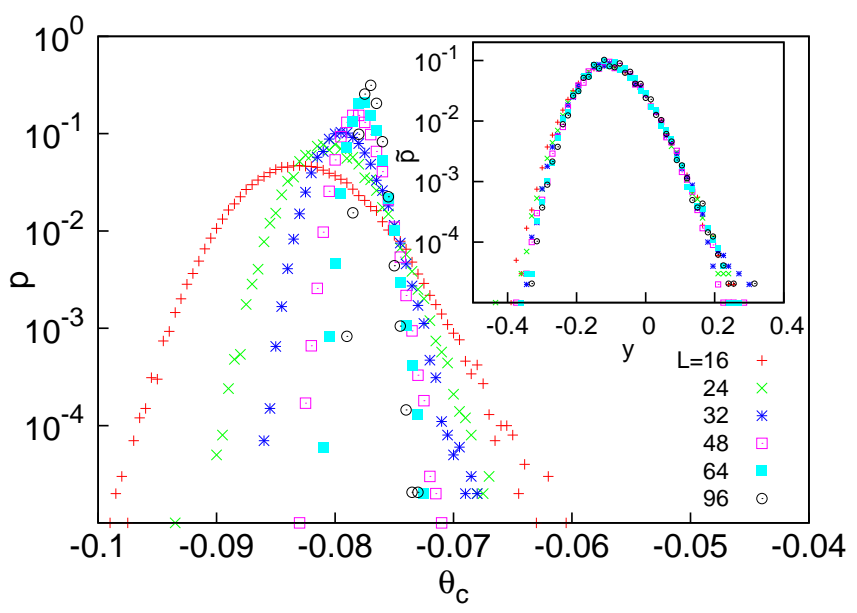

FIG. 2: (Color online) Distribution of the pseudo-critical points, $\theta_{c}(\alpha, N)$, for various sizes, $N=L^{3}$, for fixed- $h$ randomness for the $3 \mathrm{D}$ mode ${ }^{30}$. In the inset the scaled distributions are shown as a function of $y=\left(\theta_{c}(\alpha, N)-\theta_{c}\right) N^{1 / d \nu}$, see the text.

In the second step we have studied the size-dependence of the distributions of the pseudo-critical points, which is illustrated in Fig 2 for the 3D model. From the scaling of the width, $\Delta \theta_{c}(N) \sim N^{-1 / d \nu_{w}}$, and from the scaling of the mean value: $\left|\theta_{c}-\overline{\theta_{c}(N)}\right| \sim N^{-1 / d \nu_{s}}$ we have obtained the critical exponents, $\nu_{w}$ and $\nu_{s}$, respectively. We have calculated size-dependent effective exponents by two-point fits (comparing the results for sizes $N$ and $N / 2^{d}$ ), which are then extrapolated. The effective exponents for the 3D model are shown in the inset of Fig 3 for the two different randomnesses. As in this example we have generally observed that the extrapolated critical exponents are universal, i.e. randomness independent. Estimates of the exponents are presented in Table \ together with the values of the true critical points, $\theta_{c}^{(b)}$ and $\theta_{c}^{(f)}$, for the two randomnesses, respectively. One can notice in this Table that the error of the estimates is increasing with the dimensionality and the finite-size corrections are considerably strong for $4 \mathrm{D}$ and for $\mathrm{ER}$ random graphs.

Having accurate estimates for the critical points we have renormalized the systems at $\theta_{c}$ and studied the scaling behavior of the moment of the correlation cluster, $\mu(\alpha, N)$, as well as that of the log-energy parameter, $\gamma(\alpha, N)=-\ln \epsilon(\alpha, N)$. The average moment is found to scale as: $\bar{\mu}(N) \sim N^{d_{f} / d}$, where $d_{f}$ is the fractal dimension of the correlation cluster. We illustrate this relation in Fig 3 for the 3D and the 4D models, in which $\bar{\mu}(N)$ is shown as a function of $N$ in a log-log scale. Indeed, for not too small systems, $N>1000$, the points are very well on straight lines, the slope of which being the same for the two different randomnesses for the same $d$. From the cluster moment the magnetization is calculated as, $m=\bar{\mu}(N) / N$, thus we have the scaling relations: $x / d=\beta /(d \nu)=1-d_{f} / d$, where $\beta$ is the

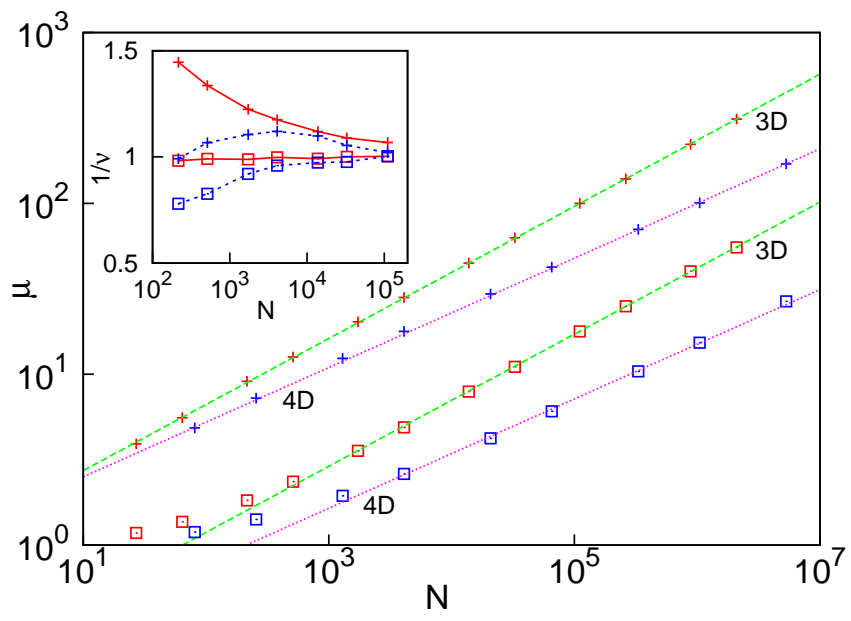

FIG. 3: (Color online) Average moment of the correlation cluster at the critical point vs. the size of the system in a loglog plot for the 3D and 4D models for two types of randomness (box- $h$ : $\square$, fixed- $h$ : + ). The slope of the straight lines is given by: $d_{f} / d=0.387$ and $d_{f} / d=0.32$ for $3 \mathrm{D}$ and $4 \mathrm{D}$, respectively. Inset: Finite-size effective exponents, $\nu_{s}$ (blue dashed) and $\nu_{w}$ (red - full), for the 3D model for two types of randomness. In all cases the error of the calculation is smaller than the size of the symbol.

magnetization exponent and $x=\beta / \nu$. Estimates for the exponents $x / d$, which are calculated through two-point fits are shown in Table 【
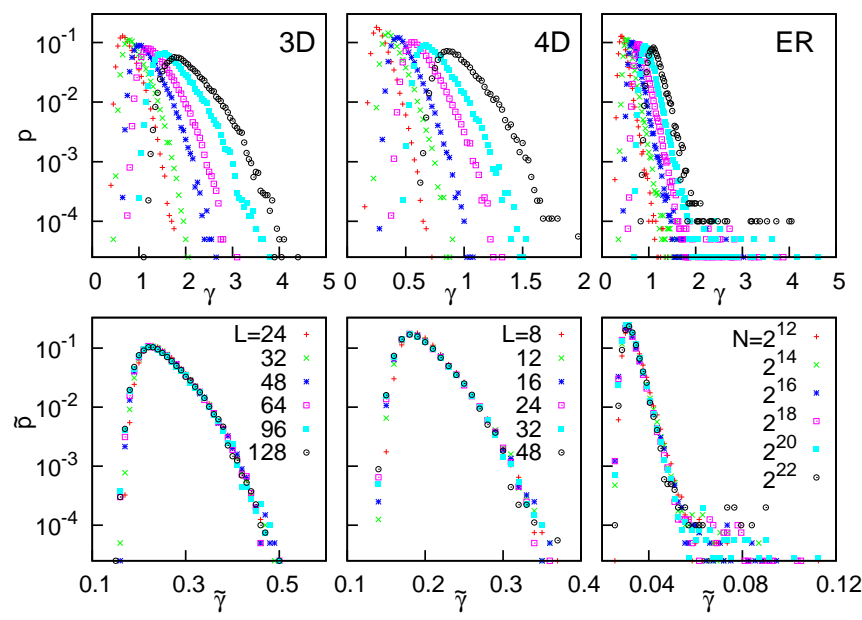

FIG. 4: (Color online) Distribution of the log-energy parameters at the critical point in $3 \mathrm{D}, 4 \mathrm{D}$ and in the ER random graph for fixed- $h$ randomness and for different sizes (upper panel $)^{30}$. In the lower panel the scaled distributions are shown, as described in the text. The constants in the scaling forms are $\gamma_{0}=-0.33(3 \mathrm{D}), \gamma_{0}=-0.23(4 \mathrm{D})$ and $\gamma_{0}=-0.5$, $\ln N_{0}=-5$. (ER).

The energy parameter, $\epsilon(\alpha, N)$, is given by the value of the smallest effective transverse field, not considering that of the correlation cluster (if any). The distribution of the log-energy parameter, $\gamma(\alpha, N)$, is shown in the upper panel of Fig 4 for the different models. As a clear 
TABLE I: Critical properties of the RTIM in three and four dimensions and in ER random graphs. $N_{\max }$ denotes the number of spins in the largest finite systems used in the RG calculation. In the last four lines by estimating the different critical exponents results obtained by the two forms of disorder are taken into account.

\begin{tabular}{|c|c|c|c|}
\hline & $3 \mathrm{D}$ & $4 \mathrm{D}$ & $\mathrm{ER}$ \\
\hline$N_{\max }$ & $128^{3}$ & $48^{4}$ & $2^{22}$ \\
\hline$\theta_{c}^{(b)}$ & $2.5305(10)$ & $3.110(5)$ & $2.775(2)$ \\
$\theta_{c}^{(f)}$ & $-0.07627(2)$ & $-0.04698(10)$ & $-0.093(1)$ \\
\hline$d \nu_{w}$ & $2.90(15)$ & $3.30(15)$ & $7 .(2)$ \\
$d \nu_{s}$ & $2.96(5)$ & $2.96(15)$ & $5 .(1)$ \\
\hline$x / d$ & $0.613(5)$ & $0.68(3)$ & $0.81(4)$ \\
\hline$\psi$ & $0.46(2)$ & $0.46(2)$ & - \\
\hline
\end{tabular}

indication of infinite disorder scaling the width of the distribution is increasing with $N$. In 3D and 4D the appropriate scaling variable is $\tilde{\gamma}=\left(\gamma(N)-\gamma_{0}\right) N^{-\psi / d}\left(\gamma_{0}\right.$ is a constant), as illustrated with the data collapse in the lower panel of Fig 4 The critical exponent $\psi$ has been calculated from the optimal collapse of the distributions, as well as from two-point fits by comparing the mean values $\bar{\gamma}(N)$ and $\bar{\gamma}\left(N / 2^{d}\right)$, which are presented in Table \. The ER random graphs are infinite dimensional objects and in this case the broadening of the distribution of the log-energy parameter is found to scale with $\ln N$. As a good scaling combination we have here $\tilde{\gamma}=\left(\gamma(N)-\gamma_{0}\right)\left(\ln N / N_{0}\right)^{-\omega}\left(\gamma_{0}\right.$ and $N_{0}$ are constants $)$, which is illustrated with the data collapse in the lower panel of Fig 4 with an exponent $\omega=1.3(2)$. Thus the width of the distribution increases somewhat faster than linear in $\ln N$, which fact justifies that also for ER random graphs the critical behavior of the RTIM is controlled by a logarithmically infinite disorder fixed point.

\section{DISCUSSION}

Our numerical RG results indicate that the critical behavior of the random transverse-field Ising model in three and four dimensions as well as in the ER random graph is controlled by infinite disorder fixed points. This fact justifies the use of the SDRG method and ensures that the calculated numerical results about the critical exponents tend to be asymptotically correct for large sizes. Since the ER random graph represents the large-dimensional limit of the problem, infinite disorder scaling is expected to be valid at any dimensions. The critical exponents presented in Table I are found to be the same for the two types of ferromagnetic disorder used in our numerical study. We expect therefore that the IDFP-s are attractive, at least for strong enough disorder. (For the weak-disorder behavior of the systems we can not make any definite statement using the SDRG method.) We note also that at the IDFP frustration does not matter, thus quantum spinglass and random quantum ferromag- net has the same infinite disorder fixed point.

Singularities of the thermodynamic quantities at small temperatures involve the exponents in Table [. For example the susceptibility and the specific heat behave as: $\chi(T) \sim(\log T)^{(d-2 x) / \psi} / T$ and $C_{V}(T) \sim(\log T)^{d / \psi}$, respectively 11,24 . The analogous expressions for the ER random graph are: $\log [T \chi(T)] \sim(1-2 x / d)(\log T)^{1 / \omega}$ and $\log \left[C_{V}(T)\right] \sim(\log T)^{1 / \omega}$.

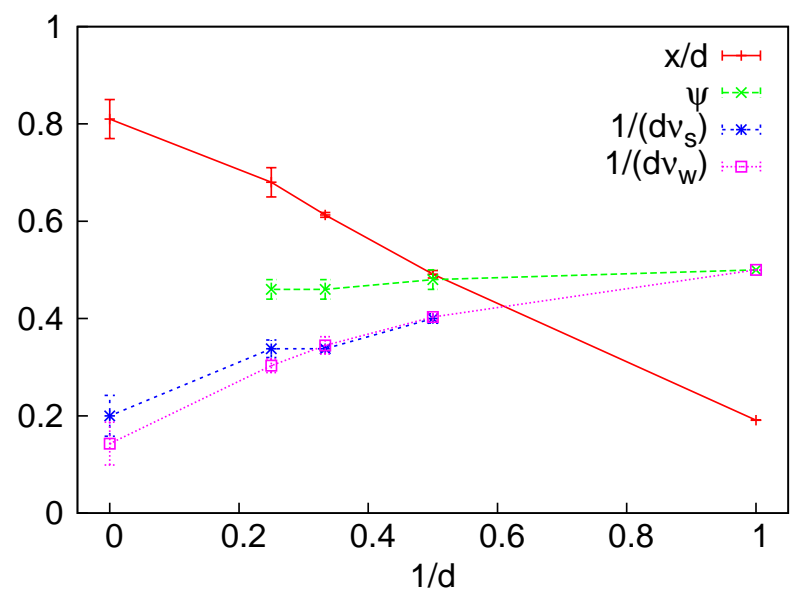

FIG. 5: (Color online) Critical exponents of the RTIM as a function of $1 / d$. At $1 / d=0$ there are results of the ER random graph.

As mentioned before the IDFP-s in Table 1 control the critical behavior of the spinglass transition, as well as that of a class of random quantum systems having an order parameter with discrete symmetry, such as random quantum Potts 31 and clock models 32 . Nonequilibrium phase transitions, such as the contact process with (strong) disorder ${ }^{21}$ also belong to this class of universality. The critical exponents in Table@ extending with the known results in $1 \mathrm{D}^{\frac{5}{4}}$ and $2 \mathrm{D}^{18}$, show a smooth variation with the dimension, which is presented in Fig 5. These results indicate that the large- $d$ limit of the problem is not singular. For a given $d$ the correlation length critical exponents, $\nu_{s}$ and $\nu_{w}$, agree with each other, within the error of the calculation ${ }^{33}$. These satisfy the rigorous bound ${ }^{34}, \nu \geq 2 / d$, and are in agreement with the scaling theory at conventional random fixed points 35,36 . Interestingly, the exponent $\psi$ is found very close to $1 / 2$ for any considered finite dimension ${ }^{37}$. This fact can be explained with our observation, that the low-energy excitations in any dimension are quasi-1D objects and the energy scale can be obtained by renormalizing these objects practically independently of the rest of the system. This leads to approximately the same type of linear-size dependence of the energy in any dimension.

At this point we want to mention a very recent study by Dimitrova and Mézard ${ }^{38}$ on the critical behavior of the RTIM by the cavity method ${ }^{39}$. In $1 \mathrm{D}$ even the simple mean-field cavity method is shown to recover some of the exact results, such as infinite disorder scaling at the critical point. On the contrary, results on the Bethe 
lattice indicate the presence of a conventional random fixed point with a finite dynamical exponent. This result is probably due to the fact, that the local topology of the Bethe lattice is different from that of the hypercubic lattices, we considered in this paper. The local topology has already been found to have an important effect on the critical behavior of random quantum systems $\stackrel{40}{\underline{4}}$. For example in the Bethe lattice it seems to be impossible to define an isotropic and quasi-one-dimensional cluster, which could be relevant for the low-energy excitations and thus for infinite disorder scaling. A direct SDRG study of the Bethe lattice RTIM could clarify some of the open questions.

Finally we note, that the SDRG investigations presented in this paper can be extended in several direc- tions. Here we mention the calculation of the entanglement entropy $15-17,41$ in these systems, as well as study of the dynamical singularities in the disordered and ordered Griffiths phases²4.

\section{Acknowledgments}

This work has been supported by the Hungarian National Research Fund under grant No OTKA K62588, K75324 and K77629 and by a German-Hungarian exchange program (DFG-MTA). We are grateful to D. Huse for helpful correspondence and suggestions and to $\mathrm{P}$. Szépfalusy and H. Rieger for useful discussions.
* Electronic address: ikovacs@szfki.hu

† Electronic address: igloi@szfki.hu

1 S. Sachdev, Quantum Phase Transitions (Cambridge University Press, 1999)

2 For reviews, see: H. Rieger and A. P Young, in Complex Behavior of Glassy Systems, ed. M. Rubi and C. PerezVicente, Lecture Notes in Physics 492, p. 256, SpringerVerlag, Heidelberg, 1997; R. N. Bhatt, in Spin glasses and random fields A. P. Young Ed., World Scientific (Singapore, 1998).

3 D.H. Reich et al., Phys. Rev. B42, 4631 (1990); W. Wu et al., Phys. Rev. Lett. 67, 2076 (1991); W. Wu et al., Phys. Rev. Lett. 71, 1919 (1993); J. Brooke et al., Science 284, 779 (1999).

4 S. M. A. Tabei, et al., Phys. Rev. Lett. 97, 237203 (2006); M. Schechter, Phys. Rev. B 77 020401(R) (2008); M. Schechter M. and P. C. E. Stamp, EPL 88, 66002 (2009).

5 D.S. Fisher, Phys. Rev. Lett. 69, 534 (1992); Phys. Rev. B 51, 6411 (1995).

${ }^{6}$ F. Iglói, Phys. Rev. B65, 064416 (2002).

7 B. M. McCoy and T. T. Wu, Phys. Rev. 176, 631 (1968); Phys. Rev. 188, 982 (1969); B. M. McCoy, Phys. Rev. 188, 1014 (1969); Phys. Rev. B 2, 2795 (1970).

8 R. Shankar and G. Murthy, Phys. Rev. B 36, 536 (1987).

9 A. P. Young and H. Rieger, Phys. Rev. B 53, 8486 (1996).

${ }^{10}$ F. Iglói and H. Rieger, Phys. Rev. B57 11404 (1998).

11 D.S. Fisher, Physica A 263, 222 (1999)

12 O. Motrunich, S.-C. Mau, D.A. Huse and D.S. Fisher, Phys. Rev. B 61, 1160 (2000).

13 Y.-C. Lin, N. Kawashima, F. Iglói and H. Rieger, Progress in Theor. Phys. 138, (Suppl.) 479 (2000).

14 D. Karevski, et al., Eur. Phys. J. B 20267 (2001).

15 Y-C. Lin, F. Iglói and H. Rieger, Phys. Rev. Lett. 99, 147202 (2007).

16 R. Yu, H. Saleur and S. Haas, Phys. Rev. B 77, 140402 (2008).

17 I. A. Kovács and F. Iglói, Phys. Rev. B 80, 214416 (2009).

18 I. A. Kovács and F. Iglói, Phys. Rev. B 82, 054437 (2010).

19 C. Pich, A.P. Young, H. Rieger and N. Kawashima, Phys. Rev. Lett. 81, 5916 (1998).

20 T. Vojta, A. Farquhar and J. Mast, Phys. Rev. E 79, 011111 (2009).

21 J. Hooyberghs, F. Iglói and C. Vanderzande, Phys. Rev.
Lett. 90 100601, (2003); Phys. Rev. E 69, 066140 (2004).

22 P. B. Chakraborty, P. Henelius, H. Kjønsberg, A. W. Sandvik, and S. M. Girvin, Phys. Rev. B 70, 144411 (2004).

23 P. Erdős, and A. Rényi, Publicationes Mathematicae 6, 290 (1959).

${ }^{24}$ For a review, see: F. Iglói and C. Monthus, Physics Reports 412, 277, (2005).

25 S.K. Ma, C. Dasgupta and C.-K. Hu, Phys. Rev. Lett. 43, 1434 (1979); C. Dasgupta and S.K. Ma, Phys. Rev. B22, 1305 (1980).

26 The algorithm described in Ref ${ }^{18}$ has the same performance in $2 \mathrm{D}$, but it is considerably slower in higher dimensions.

27 To select site $j$ we first identify a non-decimated end-site to $j$, which is denoted by $k(j)$. Performing the renormalization around $j$ several nearby sites (including $j$ ) could be eliminated and $k(j)$ is one such site, which is not eliminated directly. We measure the effective coupling between $i$ and $k(j)$ as $J_{i, k(j)}$ and its maximal value for different $k(j)$-s is $\tilde{J}_{i, j}$. Now the relevant site to $i$ is such $j$, for which $\tilde{J}_{i, j}$ is maximal.

28 This type of disorder could be relevant for experimental realizations, in which the transverse field is homogeneous, see c.f. $\mathrm{LiHo}_{\mathrm{x}} \mathrm{Y}_{1-\mathrm{x}} \mathrm{F}_{4}$.

29 Between the copies of the ER random graphs we have drawn $N / 2$ random links.

30 The distributions are obtained from histograms, in which in the central part (within the width of the distribution) there are several thousands of samples. Here the error is smaller than the size of the symbol. At the tails the error is increasing, but these parts of the distribution have negligible influence on the values of the calculated critical exponents.

31 T. Senthil and S. N. Majumdar Phys. Rev. Lett. 76, 3001 (1996)

32 E. Carlon, P. Lajkó, and F. Iglói, Phys. Rev. Lett. 87, 277201 (2001)

33 In $4 \mathrm{D}$ the estimates of the two exponents seem to be at the border or slightly outside the estimated error. To decide about the agreement or disagreement of the two exponents in this case one should study even larger systems, in particular to reduce the error in the position of the true critical point. 
34 J. T. Chayes et al., Phys. Rev. Lett. 57, 299 (1986).

35 S. Wiseman and E. Domany, Phys. Rev. Lett. 81 (1998) 22; Phys Rev E 58 (1998) 2938.

36 A. Aharony, A.B. Harris and S. Wiseman, Phys. Rev. Lett. 81 (1998) 252.

${ }^{37}$ For $1 \mathrm{D}$ and $2 \mathrm{D}$ we have $\psi=0.5^{5}$ and $\psi=0.48(2)^{18}$, respectively.

38 O. Dimitrova and M. Mézard, J. Stat. Mech. P01020 (2011).
39 L. B. Ioffe and M. Mézard, Phys. Rev. Lett. 105, 037001 (2010); F. M. Veigelman, L. B. Ioffe and M. Mezard, Phys. Rev. B 82, 184534 (2010).

40 R. Mélin, B. Douçot, and F. Iglói, Phys. Rev. B 72024205 (2005).

41 G. Refael and J. E. Moore, J. Phys. A: Math. Theor. 42, 504010 (2009). 